\title{
Projeto de Estabilizadores utilizando o Grey Wolf Optimization e Validação via o Programa Simulight
}

\author{
Junior N. N. Costa. Wesley Peres. \\ Francisco C. R. Coelho. Rayane R. Leal.
}

\author{
Programa de Pós-Graduação em Engenharia Elétrica \\ (PPGEL/UFSJ/CEFET-MG), Departamento de Engenharia Elétrica \\ (DEPEL), Universidade Federal de São João del-Rei (UFSJ), São \\ João-del Rei, MG, Brasil (e-mails: juniornatanncosta@gmail.com; \\ wesley.peres@ufsj.edu.br; franciscocoelho@ufsj.edu.br; \\ rayanerayner@gmail.com).
}

\begin{abstract}
This paper deals with the coordinated design of power system stabilizers in order to add damping torque to low frequency oscillations in the South Brazilian multimachine power system. The tuning procedure is formulated as an optimization problem aiming at maximizing the damping ratio associated with the dominant eigenvalue in closed-loop operation. For this purpose, the power system linearized model has been represented in state space formulation by using the Matlab environment. The optimization problem is solved through the recently proposed Grey Wolf Optimization method whose performance is evaluated for different initial conditions. Finally, the effectiveness of the controllers is validated through nonlinear simulations by using the Simulight software.
\end{abstract}

Resumo: O presente artigo trata do projeto coordenado de estabilizadores de sistemas de potência com o objetivo de adicionar torque de amortecimento às oscilações eletromecânicas de baixa frequência no sistema multimáquinas Sul Brasileiro. O procedimento de ajuste é formulado como um problema de otimização visando à maximização do coeficiente de amortecimento do autovalor dominante em malha fechada. Para essa finalidade, o modelo linearizado do sistema de potência foi representado em espaço de estados na ferramenta Matlab. O problema de otimização é resolvido pela técnica Grey Wolf Optimization, recentemente proposta na literatura, para a qual é feita uma análise de desempenho partindo-se de diferentes condições iniciais. Para a validação dos controladores projetados, procede-se com simulações não lineares utilizando-se o software Simulight.

Keywords: Power system stabilizers; Grey Wolf Optimization; Power system stability; Power system control; Simulight.

Palavras-chaves: Estabilizadores de sistemas de potência; Grey Wolf Optimization; Estabilidade de sistemas de potência; Controle de sistemas de potência; Simulight.

\section{INTRODUÇÃO}

A estabilidade a pequenas perturbações de Sistemas Elétricos de Potência (SEP) está associada à habilidade desses sistemas alcançarem um novo ponto de equilíbrio após pequenas variações de carga e geração que ocorrem constantemente ao longo do dia. Essa habilidade está associada ao equilíbrio entre os torques mecânico e elétrico nos geradores síncronos de forma que estes voltem a operar na velocidade síncrona após os distúrbios. Conforme (Sauer \& Pai, 1998), as oscilações de velocidade e de ângulo do rotor são denominadas de oscilações eletromecânicas de baixa frequência.

Do ponto de vista de modelagem, o estudo da estabilidade a pequenas perturbações é baseado em modelos linearizados do sistema de equações algébrico-diferencial que descreve a dinâmica do sistema. A partir do modelo linearizado em espaço de estados, procede-se com a Análise Modal (análise dos modos ou autovalores) visando identificar a posição dos pólos no plano complexo, que está associada à magnitude, amortecimento, frequência e comportamento das oscilações eletromecânicas (Sauer \& Pai, 1998).

$\mathrm{Na}$ existência de autovalores complexos instáveis, o sistema estará sujeito a oscilações de amplitude crescente após a ocorrência de pequenas perturbações. Oscilações não amortecidas, fisicamente, causam desgastes mecânicos nos geradores, reduzem os limites de transferência de potência e podem causar blecautes (Kundur, 1994).

Com o objetivo de amortecer as oscilações e/ou tornar o SEP estável, procede-se com a alocação e ajuste dos Estabilizadores de Sistemas de Potência (ESP). Os ESP são controladores propostos por (Demello \& Concordia, 1969) na década de setenta e amplamente utilizados pelas empresas do setor elétrico. No Brasil, para acesso à rede básica, os geradores devem ser equipados com estabilizadores. A função do ESP é a modulação da tensão de campo e 
consequentemente da tensão e potência elétrica terminal com o objetivo de adicionar amortecimento às oscilações.

Considerando a existência de diversos estabilizadores, os seus parâmetros (blocos de ganho e de compensação de fase) devem ser ajustados de forma coordenada (ou simultânea) para se evitar interações indesejadas. Uma das vertentes utilizadas na literatura para o projeto desses controladores é a aplicação de métodos de otimização, por tornar o processo automático e prático (Dill \& e Silva, 2012).

Uma das formulações mais abordadas na literatura é a maximização do coeficiente de amortecimento (Do Bomfim et al., 2000; Abdel-Magid \& Abido, 2003; Peres et al., 2018) considerando diferentes pontos operativos para a garantia de robustez. Considerando a natureza não convexa do problema, a solução por metaheurísticas populacionais são recorrentes na literatura. Estas são baseadas na troca de informações entre os indivíduos (conhecimento coletivo) e no balanceamento entre as etapas de busca global (exploração) e local (intensificação), permitindo que soluções viáveis e de boa qualidade sejam obtidas com um custo computacional adequado. Em (Peres et al., 2018) apresenta-se uma revisão das principais metaheurísticas aplicadas para a solução desse problema: Algoritmos Genéticos, Otimização por Enxame de Partículas, Otimização baseada em Colônia de Formigas, Algoritmo baseado na Ecolocalização de Morcegos, Algoritmo baseado na Reprodução dos Cucos, Busca Gravitacional, Evolução Diferencial, entre outros.

Sabe-se que, em média, de acordo com o "The No Free Lunch Theorem for Optimization" enunciado por (Wolpert \& Macready, 1997), nenhum algoritmo é melhor do que outro. Isso tem motivado o desenvolvimento de algoritmos que forneçam soluções competitivas aos já existentes e que melhorem a qualidade da solução quando possível. Nesse contexto, (Mirjalili et al., 2014) propuseram um algoritmo baseado na hierarquia e comportamento social presente em alcateias de lobos cinzentos denominado Grey Wolf Optimization $(G W O)$. O GWO já foi aplicado em diversos problemas matemáticos e de engenharia (Hatta et al., 2018), incluindo o projeto de ESP (Shakarami \& Davoudkhani, 2016).

No presente trabalho, é proposto o projeto de ESPs do sistema Sul Brasileiro para a maximização do coeficiente de amortecimento em malha fechada via a aplicação do $G W O$. Adicionalmente, é realizada uma análise estatística dos resultados fornecidos pelo GWO. O objetivo é avaliar a robustez do método $G W O$ quanto às condições iniciais utilizadas.

Por fim, utiliza-se a versão acadêmica do programa Simulight para a validação não linear dos controladores projetados. O Simulight é um simulador desenvolvido pela COPPE/UFRJ em parceria com a concessionária Light para a análise de sistemas monofásicos e/ou trifásicos com geradores distribuídos (Assis et al., 2006). Ressalta-se que esse simulador não contém um módulo de projeto de controladores. Por isso, nesse trabalho, o sistema algébricodiferencial empregado no Simulight foi linearizado e implementado no Matlab para o projeto dos controladores. Como resultado, além da efetividade dos ESP projetados, também é mostrada a aderência entre os modelos não linear do Simulight e o modelo linearizado implementado pelos autores.

\section{APRESENTAÇÃO DO PROBLEMA}

\subsection{Modelo Algébrico-Diferencial}

A dinâmica do SEP é descrita por um sistema diferencialalgébrico não linear conforme descrito nas equações (1)-(2).

$$
\begin{aligned}
\dot{x} & =f(x, z, u) \\
0 & =g(x, z, u)
\end{aligned}
$$

onde tem-se os vetores de variáveis de estado $(x)$, algébricas $(z)$ e de entrada $(u)$. A parcela diferencial de primeira ordem (equação (1)) descreve a dinâmica das máquinas elétricas e dos dispositivos de controle. A parte algébrica (equação (2)) está associada ao balanço de potência nodal da rede, relação entre as tensões internas e terminais das máquinas elétricas e relações entre as variáveis dos controladores.

Nesse trabalho, os geradores foram representados por um modelo de terceira ordem e os reguladores de tensão por um modelo de primeira ordem (reguladores estáticos), conforme (Sauer and Pai, 1998).

Para a análise da resposta do sistema às grandes perturbações (Estabilidade Transitória), como a que será conduzida via o Simulight para validação dos controladores projetados, o sistema de equações (1)-(2) é integrado via o método Trapezoidal Implícito (simulação no domínio do tempo).

Para a análise da resposta a pequenos distúrbios (Estabilidade a Pequenas Perturbações) e o projeto dos estabilizadores, o sistema de equações (1)-(2) será linearizado e representado em espaço de estados utilizando o Matlab. Ressalta-se que o modelo linearizado é válido para pequenos distúrbios ao redor do ponto utilizado no processo de linearização (Kundur, 1994).

\subsection{Modelo do Sistema em Malha Aberta (sem ESP)}

A equação (3) descreve o sistema linearizado (em espaço de estados) em malha aberta.

$$
\begin{aligned}
& \dot{x}=A \cdot x+B \cdot u \\
& y=C \cdot x+D \cdot u
\end{aligned}
$$

em que, novamente, $x$ são as variáveis de estado (tensões internas de eixo d, velocidades, ângulos e tensões de campo), $u$ representa as variáveis de entrada (tensão de referência do regulador de tensão) e $y$ são as variáveis de saída (velocidade angular).

Em malha aberta, os geradores possuem apenas os reguladores automáticos de tensão. Portanto, o sistema não possui estabilizadores.

\subsection{Sistema em Malha Fechada (com ESP)}

Após a inclusão dos controladores através de um processo de realimentação, o modelo em espaço de estados é obtido conforme a equação (4). 


$$
\begin{gathered}
x_{\dot{M F}}=A_{M F} \cdot x_{M F}+B_{M F} \cdot u \\
y=C_{M F} \cdot x_{M F}+D_{M F} \cdot u
\end{gathered}
$$

em que $x_{M F}$ é o vetor de variáveis de estado em malha fechada que, além das variáveis do sistema em malha aberta ( $x$ na equação (3)), também inclui as variáveis de estado dos controladores.

A estabilidade do sistema em malha fechada pode ser avaliada a partir dos autovalores de $A_{M F}$ (Kundur, 1994; Sauer \& Pai, 1998).

\subsection{Estrutura do Controlador}

O processo de realimentação é feito conforme a Fig. 1.

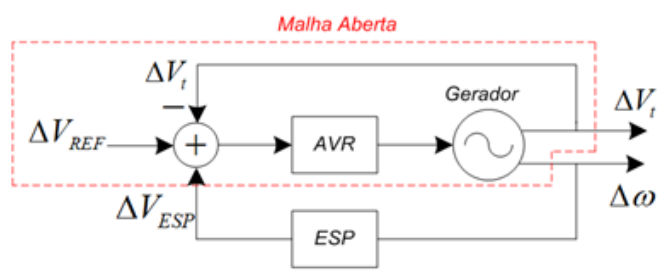

Fig. 1 Sistema em malha fechada.

A estrutura clássica do controlador utilizada no trabalho é apresentada na equação (5).

$$
E S P(s)=K_{E S P} \cdot\left(\frac{1+s T_{1}}{1+s T_{2}}\right) \cdot \frac{s T_{w}}{1+s T_{w}}
$$

em que os parâmetros ajustáveis são $K_{E S P}, T_{1}$ e $T_{2}$. O filtro washout possui a finalidade de anular o sinal de saída do ESP em regime permanente e sua constante $T_{w}$ não é crítica e foi escolhida com base na literatura (3 segundos).

\subsection{Problema de Otimização}

O problema de ajuste é formulado como um problema de otimização com o objetivo de maximizar o coeficiente de amortecimento dos autovalores do sistema em malha fechada.

A função objetivo do problema, definida na equação (6), corresponde ao mínimo amortecimento $\xi_{\min }$ obtido considerando todos os autovalores em malha fechada.

$$
\left\{\begin{array}{cc}
\text { Max } & \xi_{\min } \\
& K_{E S P \min } \leq K_{E S P} \leq K_{E S P \max } \\
\text { sa } & T_{1 \min } \leq T_{1} \leq T_{1 \max } \\
& T_{2 \min } \leq T_{2} \leq T_{2 \max }
\end{array}\right.
$$

em que $\xi$ denota o coeficiente de amortecimento de um determinado autovalor complexo $\lambda=\sigma \pm j \omega$.

A estrutura de uma solução $\operatorname{sol}_{i}$ é ilustrada na equação (7), para um caso ilustrativo de 2 geradores (2 ESPs).

$$
\operatorname{sol}_{i}=\left[\begin{array}{llllll}
K_{E S P 1} & K_{E S P 2} & T_{11} & T_{12} & T_{21} & T_{22}
\end{array}\right]
$$

\section{GREY WOLF OPTIMIZATION}

O Grey Wolf Optimization é um método de otimização baseado nas técnicas de caça e hierarquia social dos lobos cinzentos (Canis Lupus), pertencentes à família Canidae.
Proposto por (Mirjalili et al., 2014), se mostrou altamente competitivo quando comparado a outras técnicas bastante consolidadas na literatura como o Particle Swarm Optmization (PSO).

Os lobos cinzentos adotam uma hierarquia bem definida e restrita. O líder é chamado de alpha $(\alpha)$ e é o responsável pela tomada de decisões. Curiosamente ele não é o mais forte, mas o melhor em termos de gerenciamento, mostrando que a organização e a disciplina de uma alcateia é mais importante que sua força. No segundo nível hierárquico estão os chamados betas $(\beta)$, conselheiros do alpha $\mathrm{e}$ disciplinadores dos níveis inferiores. O terceiro nível é ocupado pelos deltas $(\delta)$, encarregados da segurança da alcateia. Por fim, estão os ômegas $(\omega)$, uma espécie de "bode expiatório" do grupo, obrigados a servir os demais níveis.

Além da hierarquia, a caça em um grupo de lobos cinzentos é outro comportamento social interessante. Ela pode ser dividida em:

- $\quad$ Cercando a presa - durante a caça os lobos cercam a presa. A modelagem desse comportamento é feita através equações (8) a (11):

$$
\begin{gathered}
\vec{D}=\left|\vec{C} \cdot \vec{X}_{p}(t)-\vec{X}(t)\right| \\
\vec{X}(t+1)=\vec{X}_{p}(t)-\vec{A} \cdot \vec{D} \\
\vec{A}=2 \vec{a} \cdot \vec{r}_{1}-\vec{a} \\
\vec{C}=2 \cdot \vec{r}_{2}
\end{gathered}
$$

em que $t$ é a iteração atual, $\vec{A}$ e $\vec{C}$ são coeficientes vetoriais, $\vec{X}_{p}$ é o vetor de posição da presa, $\vec{X}$ é o vetor de posição do lobo, $\vec{r}_{1}$ e $\vec{r}_{2}$ são vetores randômicos no intervalo de $[0,1]$ e $\vec{a}$ é um vetor linearmente diminuído de 2 à 0 no decorrer das iterações. A dimensão de cada indivíduo (lobo) foi ilustrada na equação (7) para o caso de dois estabilizadores;

Caçando a presa - os lobos possuem a capacidade de reconhecer a localização da presa e envolvê-la. No entanto, matematicamente, não se conhece $\vec{X}_{p}$. Para contornar esse problema, considera-se que alpha, beta e delta possuem o melhor conhecimento sobre a potencial localização da presa, ou seja, estima-se que ela esteja próxima das três melhores soluções. Assim, obriga-se os demais (ômegas) a se posicionarem de acordo com essa estimativa. As equações de (12) a (14) modelam esse comportamento.

$$
\begin{gathered}
\left\{\begin{array}{l}
\vec{D}_{\alpha}=\left|\vec{C}_{1} \cdot \vec{X}_{\alpha}-\vec{X}\right| \\
\vec{D}_{\beta}=\left|\vec{C}_{2} \cdot \vec{X}_{\beta}-\vec{X}\right| \\
\vec{D}_{\delta}=\left|\vec{C}_{3} \cdot \vec{X}_{\delta}-\vec{X}\right|
\end{array}\right. \\
\left\{\begin{array}{l}
\vec{X}_{1}=\vec{X}_{\alpha}-\vec{A}_{1} \cdot \vec{D}_{\alpha} \\
\vec{X}_{2}=\vec{X}_{\beta}-\vec{A}_{2} \cdot \vec{D}_{\beta} \\
\vec{X}_{3}=\vec{X}_{\delta}-\vec{A}_{3} \cdot \vec{D}_{\delta}
\end{array}\right. \\
\vec{X}(t+1)=\frac{\vec{X}_{1}+\vec{X}_{2}+\vec{X}_{3}}{3}
\end{gathered}
$$

- $\quad$ Atacando a presa - os lobos terminam a caça quando a presa para de se mover. Para modelar essa aproximação à presa, diminui-se linearmente o vetor $\vec{a}$. Com 
isso, o vetor $\vec{A}$, aleatório no intervalo de [-2a 2a] também é diminuído. Se o $|\vec{A}|<1$, as soluções convergem para aquela solução, do contrário, divergem daquela solução.

Vale mencionar que o vetor $\vec{C}$ não é linearmente diminuído como acontece com $\vec{A}$, porque é importante que esse vetor forneça valores aleatórios em todos os momentos para ressaltar a exploração do espaço de busca tanto nas iterações iniciais quanto nas iterações finais. Esse componente é extremamente útil no caso de problemas envolvendo ótimos locais.

O algoritmo básico do GWO é apresentado na Fig. 2.

\begin{tabular}{|c|c|}
\hline Algoritmo $G W O$ & $\underline{\text { Passo }}$ \\
\hline Início & 1 \\
\hline $\begin{array}{l}\text { Inicializar a população de lobos cinzentos } \\
X_{i}(i=1,2, \ldots, n)\end{array}$ & 2 \\
\hline Inicializar $a, A$ e $C$ & 3 \\
\hline Calcular a aptidão de cada indivíduo & 4 \\
\hline$X_{\alpha} \leftarrow$ o melhor indivíduo & 5 \\
\hline$X_{\beta} \leftarrow$ o segundo melhor indivíduo & 6 \\
\hline$X_{\delta} \leftarrow$ o terceiro melhor indivíduo & 7 \\
\hline Inicializar o contador de iterações $t \leftarrow 1$ & 8 \\
\hline $\begin{array}{l}\text { Enquanto }(t<\text { número máximo de iterações) } \\
\text { Faça }\end{array}$ & 9 \\
\hline Para cada indivíduo Faça & 10 \\
\hline $\begin{array}{l}\text { Atualizar as posições dos indivíduos } \\
\text { usando a equação (14) }\end{array}$ & 11 \\
\hline Fim-Para & 12 \\
\hline Atualizar $a, A$ e $C$ & 13 \\
\hline Calcular a aptidão de todos os indivíduos. & 14 \\
\hline Atualizar $X_{\alpha}, X_{\beta}$ e $X_{\delta}$ & 15 \\
\hline$t \leftarrow 1+1$ & 16 \\
\hline Fim Enquanto & 17 \\
\hline Retornar $X_{\alpha}$ & 18 \\
\hline Fim & 19 \\
\hline
\end{tabular}

Fig. 2 Algoritmo básico do GWO.

Ressalta-se que cada indivíduo representa uma possível solução para o problema de ajuste de controladores, conforme ilustrado na equação (7). Com isso, no passo 14, partindo-se do sistema em malha aberta (equação (3)) calcula-se a matriz de estados em malha fechada $A_{M F}$ (equação (4)), os respectivos autovalores e coeficientes de amortecimento. O menor coeficiente de amortecimento $\xi_{\min }$ é a função aptidão.

Nos passos 2 e 11, quando se inicializa ou se procede com a atualização da posição dos indivíduos (lobos), deve-se garantir que os valores estejam dentro dos limites físicos descritos na equação (6). Na ocorrência de violações, o parâmetro é colocado no limite violado (tratamento de restrições por correção).

\section{ESTUDO DE CASO}

O método $G W O$ foi aplicado para o projeto dos controladores do sistema Sul Brasileiro, composto por 10 geradores, 45 barras e 73 linhas de transmissão. Os dados do sistema podem ser obtidos em (Menezes, 2014). Para as simulações utilizou-se a versão 2010a do Matlab e a versão acadêmica 2018 do programa Simulight.

\subsection{Descrição do Sistema}

O diagrama unifilar do sistema Sul Brasileiro é apresentado na Fig. 3.

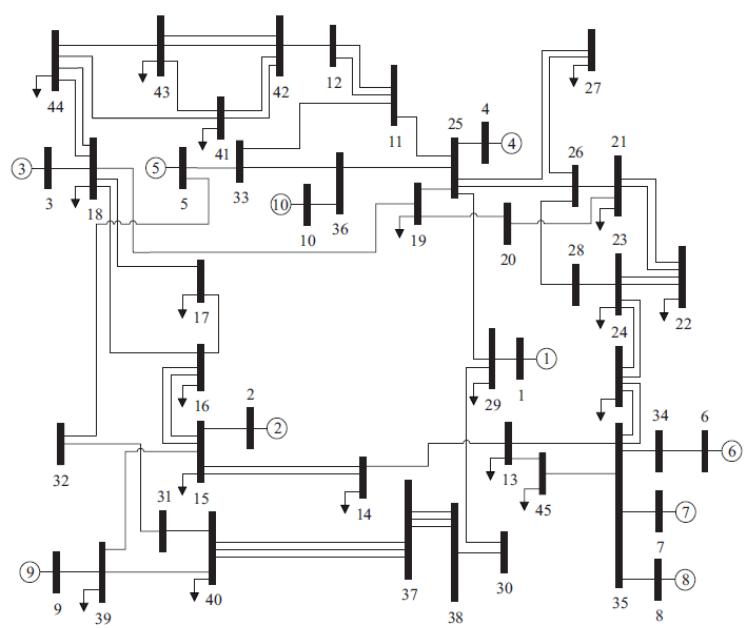

Fig. 3 Diagrama unifilar do sistema Sul Brasileiro. Fonte:

(Menezes, 2014).

Inicialmente foi conduzida uma simulação de fluxo de potência utilizando-se o Simulight e os resultados foram utilizados para a construção do modelo em espaço de estados no Matlab (modelo em espaço de estados). A análise modal, cujos resultados são apresentados na Tabela 1, indicou a existência de cinco pares de autovalores instáveis (sistema instável em malha aberta).

Tabela 1. Autovalores Dominantes do Sistema em Malha Aberta.

\begin{tabular}{|c|c|c|c|}
\hline Modos & Autovalores & $\xi$ & $\begin{array}{c}\omega_{n} \\
(\mathrm{rad} / \mathrm{seg})\end{array}$ \\
\hline 1 & $0,1501 \pm \mathrm{j} 8,1282$ & $-0,0185$ & 8,13 \\
\hline 2 & $0,1027 \pm \mathrm{j} 7,3036$ & $-0,0141$ & 7,30 \\
\hline 3 & $0,0837 \pm \mathrm{j} 6,8043$ & $-0,0123$ & 6,80 \\
\hline 4 & $0,0773 \pm \mathrm{j} 6,2816$ & $-0,0123$ & 6,28 \\
\hline 5 & $0,0378 \pm \mathrm{j} 7,0909$ & $-0,0053$ & 7,09 \\
\hline 6 & $-0,0179 \pm \mathrm{j} 7,8450$ & 0,0023 & 7,84 \\
\hline 7 & $-0,0502 \pm \mathrm{j} 8,7474$ & 0,0057 & 8,75 \\
\hline 8 & $-0,1391 \pm \mathrm{j} 9,7290$ & 0,0143 & 9,73 \\
\hline 9 & $-0,2999 \pm \mathrm{j} 9,7989$ & 0,0306 & 9,80 \\
\hline
\end{tabular}

\subsection{Definição de Parâmetros}

A análise modal realizada na seção anterior indicou a necessidade da instalação e projeto de estabilizadores nos geradores. Os coeficientes de amortecimento negativos estão associados a oscilações não amortecidas de amplitude crescente na ocorrência de distúrbios na rede (pequenos ou grandes). Visando a análise do desempenho do método $G W O$ em um problema de maior porte, todos os geradores serão equipados com um estabilizador (10 estabilizadores no sistema). Com isso, tem-se um problema de otimização com 
30 parâmetros, cujos limites são apresentados na equação (15).

$$
\begin{gathered}
1,00 \leq K_{E S P} \leq 20,00 \\
0,01 \leq T_{1} \leq 1,00 \\
0,01 \leq T_{2} \leq 0,06
\end{gathered}
$$

\subsection{Análise da Robustez quanto à População Inicial}

Em se tratando de algoritmos de otimização metaheurísticos, é desejável que os mesmos sejam capazes de encontrar boas soluções para o problema de otimização com relativa independência das condições iniciais de busca (Vasant et al., 2016). Esta característica pode ser compreendida como robustez em relação à população inicial.

Para avaliação do GWO com relação a este aspecto, executaram-se simulações em duas situações distintas. Na primeira, o $G W O$ iniciou o seu processo de busca a partir de um mesmo conjunto de soluções iniciais, sendo realizadas 100 repetições. Na segunda situação, a execução repetida do GWO foi realizada, também 100 vezes, partindo de soluções iniciais diferentes e aleatórias, provenientes de uma distribuição uniforme de probabilidade.

A Fig. 4 exibe o boxplot com os resultados obtidos nas duas situações, sendo PIF - População Inicial Fixa, e PIV População Inicial Variável.

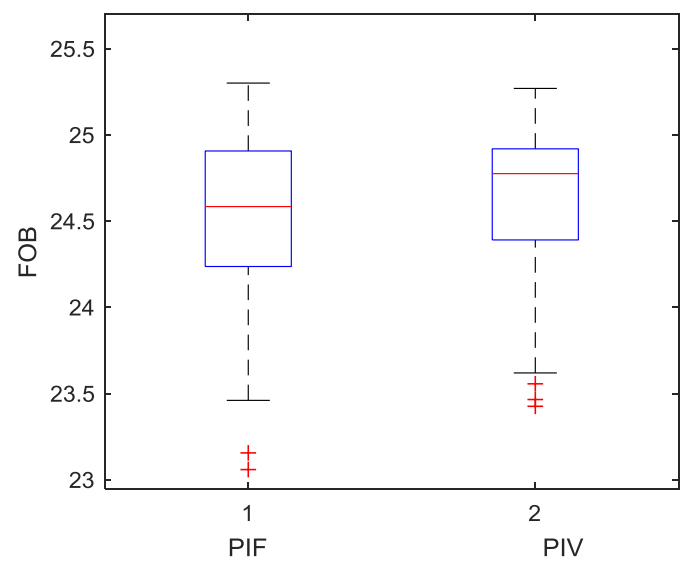

Fig. 4 Robustez do $G W O$ com relação à população inicial.

Pela análise da Fig. 4 é possível inferir que a metaheurística utilizada é capaz de produzir bons resultados com relativa independência das condições iniciais de exploração do espaço de soluções. No entanto, com o intuito de se verificar a robustez do método com rigor estatístico, realizou-se um teste de hipótese conhecido como Soma dos postos de Wilcoxon. Trata-se de um teste não paramétrico descrito em (Montgomery and Runger, 2014).

$\mathrm{O}$ teste realizado permite que se verifique a probabilidade de que as diferenças presentes em duas amostras não sejam devidas ao acaso (trata-se do valor-p). É usual o nível de significância de 5\%, que significa que se o valor- $p$ se situar abaixo de 5\%, rejeita-se a hipótese nula (que estabelece a igualdade entre as amostras, em termos de média).

Considerando então este nível de significância, ou intervalo de confiança de $95 \%$, aplicou-se o referido teste às duas amostras obtidas. O valor- $p$ encontrado ficou próximo a $9,1 \%$, não sendo possível, portanto, a rejeição da hipótese nula.

O resultado proveniente da Soma dos postos de Wilcoxon é de que não se pode afirmar que as duas amostras possuem diferenças significativas, o que é um indicativo de robustez por parte da metaheurística utilizada, com relação à população inicial.

\subsection{Parâmetros Projetados e Análise do Sistema em Malha Fechada}

Uma vez identificada a robustez do método GWO às condições iniciais utilizadas, essa seção apresenta uma das soluções obtidas. Os parâmetros dos estabilizadores são apresentados na Tabela 2, os quais permitem a estabilização do sistema Sul Brasileiro, conforme apresentado na Tabela 3.

Tabela 2. Parâmetros dos Estabilizadores.

\begin{tabular}{|c|c|c|c|}
\hline Dispositivo & $K_{\text {ESP }}$ & $T_{1}$ & $T_{2}$ \\
\hline ESP - G1 & 19,1094 & 0,0448 & 0,0435 \\
\hline ESP - G2 & 19,2529 & 0,0594 & 0,0252 \\
\hline ESP - G3 & 20,0000 & 0,0586 & 0,0243 \\
\hline ESP - G4 & 19,6422 & 0,0550 & 0,0101 \\
\hline ESP - G5 & 20,0000 & 0,0449 & 0,0126 \\
\hline ESP - G6 & 19,4400 & 0,0179 & 0,0252 \\
\hline ESP - G7 & 20,0000 & 0,0142 & 0,0143 \\
\hline ESP - G8 & 19,9204 & 0,0281 & 0,0115 \\
\hline ESP - G9 & 20,0000 & 0,0127 & 0,0272 \\
\hline ESP - G10 & 20,0000 & 0,0557 & 0,0510 \\
\hline
\end{tabular}

Tabela 3. Autovalores Dominantes do Sistema em Malha Fechada.

\begin{tabular}{|c|c|c|c|}
\hline Modos & Autovalores & $\xi$ & $\begin{array}{c}\omega_{n} \\
(\mathrm{rad} / \mathrm{seg})\end{array}$ \\
\hline 1 & $-1,61 \pm \mathrm{j} 6,24$ & 0,250 & 6,20 \\
\hline 2 & $-1,86 \pm \mathrm{j} 7,22$ & 0,249 & 7,46 \\
\hline 3 & $-1,94 \pm \mathrm{j} 7,46$ & 0,251 & 7,71 \\
\hline 4 & $-2,55 \pm \mathrm{j} 9,31$ & 0,264 & 9,65 \\
\hline 5 & $-3,04 \pm \mathrm{j} 10,10$ & 0,289 & 10,50 \\
\hline 6 & $-3,10 \pm \mathrm{j} 8,09$ & 0,358 & 8,66 \\
\hline 7 & $-3,98 \pm \mathrm{j} 14,40$ & 0,267 & 15,00 \\
\hline 8 & $-4,31 \pm \mathrm{j} 11,50$ & 0,351 & 12,30 \\
\hline 9 & $-4,59 \pm \mathrm{j} 14,80$ & 0,296 & 15,50 \\
\hline
\end{tabular}

\subsection{Validação Não Linear dos Controladores Projetados}

Com o objetivo de validar os controladores projetados, uma simulação não linear foi conduzida utilizando-se o simulador Simulight (Assis et al., 2006). Os estabilizadores foram incluídos na malha de excitação dos geradores síncronos e um curto circuito trifásico foi aplicado no meio da linha de transmissão entre as barras 6 e 34, com um tempo de eliminação de $100 \mathrm{~ms}$. A Fig. 5 apresenta a resposta da potência elétrica terminal dos geradores: observa-se a estabilização do sistema e o retorno das potências aos valores iniciais, uma vez que a topologia do sistema não foi alterada. 


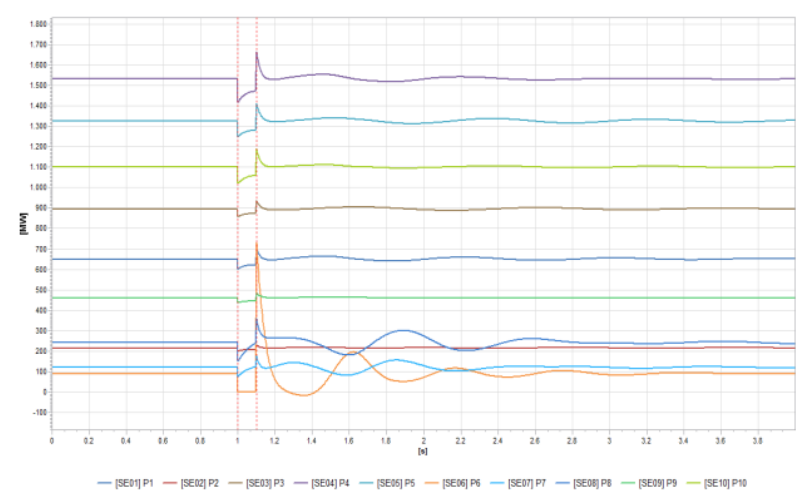

Fig. 5. Resposta da Potência Elétrica Terminal.

Com base na Fig. 5 pode-se observar que o modelo não linear do Simulight e o modelo linearizado implementado no Matlab para o projeto dos controladores são aderentes (considerando a perturbação aplicada). Ressalta-se, entretanto, que não é possível, sem uma avaliação extensiva (análise de contingências e diferentes perfis de carga e geração), afirmar que os controladores projetados sempre garantirão a estabilização do sistema na ocorrência de grandes perturbações.

\section{CONCLUSÃO}

O presente trabalho apresentou a aplicação do método Grey Wolf Optimization para o projeto coordenado de estabilizadores do sistema Sul Brasileiro, com o objetivo de se maximizar o coeficiente de amortecimento do autovalor dominante. Os seguintes pontos foram observados:

- o modelo linearizado implementado pelos autores (considerando a mesma representação de equipamentos adotada no Simulight) foi adequado para o projeto de estabilizadores e mostrou-se aderente ao modelo não linear adotado pelo Simulight (isto é, o sistema foi estabilizado em malha fechada);

- o GWO mostrou-se robusto às populações iniciais empregadas. Com base no conhecimento dos autores, essa análise ainda não tinha sido realizada na literatura e mostra que o método é adequado para o projeto de estabilizadores.

Como propostas de continuidade, são sugeridas: (i) a consideração de pontos de operação adicionais para a garantia de robustez e (ii) uma comparação com demais métodos metaheurísticos.

\section{AGRADECIMENTOS}

Os autores agradecem à FAPEMIG (projeto APQ-02245-18), CAPES (Finance Code 001), CNPq e PPGEL/UFSJ pelo auxílio financeiro. Agradecimentos também são prestados aos desenvolvedores do simulador Simulight pela disponibilização da versão acadêmica.

\section{REFERÊNCIAS}

Abdel-Magid, Y.L. and Abido, M.A. (2003) 'Optimal multiobjective design of robust power system stabilizers using genetic algorithms', IEEE Transactions on Power Systems, vol. 18, Aug, pp. 1125-1132.

Assis, T.M.L., Taranto, G.N., Falcao, D.M. and Manzoni, A. (2006) 'Long and short-term dynamic simulations in distribution networks with the presence of distributed generation', 2006 IEEE Power Engineering Society General Meeting, pp. 1-7.

Demello, F.P. and Concordia, C. (1969) 'Concepts of Synchronous Machine Stability as Affected by Excitation Control', IEEE Transactions on Power Apparatus and Systems, vol. PAS-88, April, pp. 316329.

Dill, G.K. and Silva, A.S. (2012) 'Robust Design of Power System Controllers Based on Optimization of Pseudospectral Functions', Power Systems, IEEE Transactions on, vol. 28, May, pp. 1756-1765.

Do Bomfim, A.L.B., Taranto, G.N. and Falcao, D.M. (2000) 'Simultaneous tuning of power system damping controllers using genetic algorithms', Power Systems, IEEE Transactions on, vol. 15, Feb, pp. 163-169.

Hatta, N.M., Zain, A.M., Sallehuddin, R., Shayfull, Z. and Yusoff, Y. (2018) 'Recent studies on optimisation method of Grey Wolf Optimiser (GWO): a review (2014--2017)', Artificial Intelligence Review, May.

Kundur, P. (1994) Power System Stability and Control, $1^{\text {st }}$ edition, McGraw-Hill.

Menezes, M.D. (2014) Métodos de Otimização Aplicados no Ajuste de ESPs e Controladores de Amortecimento Inseridos no FACTS-TCSC em Sistemas Elétricos de Potência (Tese de Doutorado), UNESP - Ilha Solteira.

Mirjalili, S., Mirjalili, S.M. and Lewis, A. (2014) 'Grey Wolf Optimizer', Advances in Engineering Software, vol. 69, pp. 46-61.

Montgomery, D.C. and Runger, G.C. (2014) Applied Statistics and Probability for Engineers, Sixth Edition edition, John Wiley \& Sons.

Peres, W., Silva Júnior, I.C. and Passos Filho, J.A. (2018) 'Gradient based hybrid metaheuristics for robust tuning of power system stabilizers', International Journal of Electrical Power \& Energy Systems, vol. 95, pp. 47-72.

Sauer, P. and Pai, M.A. (1998) Power System Dynamics and Stability, Prentice Hall.

Shakarami, M.R. and Davoudkhani, I.F. (2016) 'Wide-area power system stabilizer design based on Grey Wolf Optimization algorithm considering the time delay', Electric Power Systems Research, vol. 133, pp. 149159.

Vasant, P.M., Rahman, I., Singh, B.S.M. and Al-Wadud, M.A. (2016) 'Optimal power allocation scheme for plug-in hybrid electric vehicles using swarm intelligence techniques', Cogent Engineering, vol. 3, p. 1-34.

Wolpert, D.H. and Macready, W.G. (1997) 'No free lunch theorems for optimization', Evolutionary Computation, IEEE Transactions on, vol. 1, Apr, pp. 67-82. 